OPEN ACCESS

Edited by:

Ondrej Viklicky,

Institute for Clinical and Experimental

Medicine (IKEM), Czechia

Reviewed by:

Emily Thompson,

Newcastle University, United Kingdom

Burcin Ekser,

Indiana University School of Medicine,

United States

*Correspondence:

Annemarie Weissenbacher annemarie.weissenbacher@

i-med.ac.at

Specialty section: This article was submitted to

Nephrology,

a section of the journa

Frontiers in Medicine

Received: 24 October 2021 Accepted: 08 December 2021

Published: 10 January 2022

Citation:

Weissenbacher A, Stone JP, Lo

Faro ML, Hunter JP, Ploeg RJ,

Coussios CC, Fildes JE and Friend PJ (2022) Hemodynamics and Metabolic Parameters in Normothermic Kidney Preservation Are Linked With Donor Factors, Perfusate Cells, and Cytokines. Front. Med. 8:801098. doi: 10.3389/fmed.2021.801098

\section{Hemodynamics and Metabolic Parameters in Normothermic Kidney Preservation Are Linked With Donor Factors, Perfusate Cells, and Cytokines}

Annemarie Weissenbacher ${ }^{1,2 *}$, John P. Stone ${ }^{3,4}$, Maria Letizia Lo Faro ${ }^{1}$, James P. Hunter ${ }^{1}$, Rutger J. Ploeg ${ }^{1}$, Constantin C. Coussios ${ }^{5}$, James E. Fildes ${ }^{3,4}$ and Peter J. Friend ${ }^{1}$

${ }^{1}$ Oxford Transplant Centre, Nuffield Department of Surgical Sciences, University of Oxford, Oxford, United Kingdom, ${ }^{2}$ Department of Visceral, Transplant and Thoracic Surgery, Medical University of Innsbruck, Innsbruck, Austria, ${ }^{3}$ The ex-vivo Lab, Division of Cell Matrix Biology and Regenerative Medicine, School of Biological Sciences, Faculty of Biology, Medicine and Health, The University of Manchester, Manchester Academic Health Science Centre, Manchester, United Kingdom, ${ }^{4}$ The ex-vivo Research Centre Community Interest Company (CIC), Macclesfield, United Kingdom, ${ }^{5}$ Institute of Biomedical Engineering, University of Oxford, Oxford, United Kingdom

Kidney transplantation is the best renal-replacement option for most patients with end-stage renal disease. Normothermic machine preservation (NMP) of the kidney has been studied extensively during the last two decades and implemented in clinical trials. Biomarker research led to success in identifying molecules with diagnostic, predictive and therapeutic properties in chronic kidney disease. However, perfusate biomarkers and potential predictive mechanisms in NMP have not been identified yet. Twelve discarded human kidneys ( $n=7 \mathrm{DBD}, n=5 \mathrm{DCD}$ ) underwent NMP for up to $24 \mathrm{~h}$. Eight were perfused applying urine recirculation (URC), four with replacement of urine (UR) using Ringer's lactate. The aim of our study was to investigate biomarkers (NGAL, KIM-1, and L-FABP), cells and cytokines in the perfusate in context with donor characteristics, perfusate hemodynamics and metabolic parameters. Cold ischemia time did not correlate with any of the markers. Perfusates of DBD kidneys had a significantly lower number of leukocytes after $6 \mathrm{~h}$ of NMP compared to DCD. Arterial flow, pH, NGAL and L-FABP correlated with donor creatinine and eGFR. Arterial flow was higher in kidneys with lower perfusate lactate. Perfusate TNF- $\alpha$ was higher in kidneys with lower arterial flow. The cytokines IL-1 $\beta$ and GM-CSF decreased during $6 \mathrm{~h}$ of NMP. Kidneys with more urine output had lower perfusate KIM-1 levels. Median and 6-h values of lactate, arterial flow, $\mathrm{pH}, \mathrm{NGAL}, \mathrm{KIM}-1$, and L-FABP correlated with each other indicating a 6-h period being applicable for kidney viability assessment. The study results demonstrate a comparable cytokine and cell profile in perfusates with URC and UR. In conclusion, clinically available perfusate and hemodynamic parameters correlate well with donor characteristics and measured biomarkers in a discarded human NMP model.

Keywords: kidney transplantation, organ preservation, normothermic, urine recirculation, ex-situ perfusion 


\section{INTRODUCTION}

Improving the quality and duration of donor kidney preservation prior to transplant may increase utility and potentially improve outcomes. Machine perfusion is at the forefront of this field (1-4), but accurate monitoring and evaluation of the kidney is essential to optimize outcomes. Currently, no biomarkers exist that can predict the usability or quality of a kidney during perfusion. Broad hemodynamic parameters such as arterial flow during hypothermic machine perfusion (HMP) with DGF and higher levels of NGAL and/or L-FABP over time have been inversely associated with estimated glomerular filtration rate (eGFR) together with an increasing intrarenal resistance (IRR) (5). Critical criteria consisting of arterial flow, macroscopic appearance of the kidney and volume of excreted urine have been incorporated into a decision-making score during normothermic machine perfusion (NMP) (6), but clearly, a prognostic marker of post-transplant function would represent a stepwise improvement to perfusion.

The kidney is equipped with a sophisticated immune compartment, hosting a plethora of non-hematopoetic cell types and a variety of both transient and resident leukocytes $(7,8)$. This leukocyte population remains in a steady state until an immunological challenge occurs. In the transplant setting this consists of death in the donor, surgical intervention in the donor, preservation, and finally reperfusion in the recipient. A potent inflammatory cascade ensues involving severe cytokine activation and cellular extravasation (9-11). Given this inflammatory response is initiated in the donor and continues throughout preservation, evaluating leukocyte diapedesis and cytokine secretion may identify novel biomarkers during kidney perfusion.

On these grounds, the aim of this study was to investigate if perfusion inflammatory profiles correlate with (i) donor factors, (ii) perfusion hemodynamics, (iii) the type of volume management, applying either urine recirculation (URC) or urine replacement (UR), and (iv) biomarkers of renal injury (NGAL, KIM-1, and L-FABP) in a blood-based perfusate of long-term NMP $(12,13)$ of discarded human kidneys.

\section{MATERIALS AND METHODS Donor Factors}

Human kidney grafts, deemed not transplantable by all kidney transplant centers in the United Kingdom were included in this study. All organs were retrieved for the purpose of transplantation but discarded during post-procurement assessment. Donor and retrieval characteristics, kidney function parameter, and ischemia times were collected.

Abbreviations: CIT, cold ischemia time; DBD, donation after brain death; DCD, donation after circulatory death; $\Delta$, delta; ECD, extended criteria donor; ECMO, extracorporeal membrane oxygenation; ESRD, end stage renal disease; HMP, hypothermic machine perfusion; IRR, intrarenal resistance; KIM-1, kidney injury molecule-1; LDH, lactate dehydrogenase; L-FABP, liver-type fatty acid-binding protein; NADH, nicotinamide adenine dinucleotide; NGAL, neutrophil gelatinaseassociated lipocalin; NMP, normothermic machine perfusion; SCS, static cold storage; WIT, warm ischemia time.

\section{NMP Perfusion}

After being sent to Oxford, perfusions were performed at the Institute of Biomedical Engineering, University of Oxford. The technique of NMP using discarded human kidneys was reported previously $(12,13)$. Briefly, hemodynamic (arterial blood flow, mean arterial pressure and IRR) and biochemical perfusion parameters were recorded. Perfusate samples were collected and processed by centrifugation at $4,000 \mathrm{rpm}$ for $15 \mathrm{~min}$ at $4^{\circ} \mathrm{C}$. The supernatant was aliquoted, snap-frozen and stored at $-80^{\circ} \mathrm{C}$. The study was evaluated and approved by the National Ethics Review Committee of the United Kingdom (REC reference 12/EE/0273 IRAS project ID 106793).

\section{Volume Management}

Continuous urine recirculation or urine replacement with Ringer's lactate was applied as reported previously $(13,14)$. Ringer's lactate was infused to replace the excreted urine as a 1:1 volume replenishment in $20 \mathrm{~mL}$ intervals (14). The $\mathrm{pH}$ was adjusted through titration with sodium bicarbonate 8.4\% (5$15 \mathrm{~mL}$ ) to the physiological level of 7.3 before kidney NMP was started. No additional sodium bicarbonate was given at any point during perfusion after kidney connection.

\section{Biomarkers of Renal Injury}

Neutrophil gelatinase-associated lipocalin (NGAL), kidney injury molecule-1 (KIM-1), and liver fatty acid-binding protein (L-FABP) levels in the perfusate samples were measured. Neutrophil gelatinase-associated lipocalin (NGAL), kidney injury molecule-1 (KIM-1), and liver-type fatty acidbinding protein (L-FABP) levels in the perfusate samples were measured by a quantitative sandwich enzyme immunoassay technique using NGAL and KIM-1 Quantikine ELISA kits (R\&D systems, USA) and Human FABP1/L-FABP ELISA Kit (CMIC Co., Ltd., supplied by R\&D systems) according to manufacturers' instructions (12).

\section{Inflammatory Profiling Luminex ${ }^{\circledR}$ Analysis}

A commercially available human 13-plex magnetic bead panel (Merck Millipore, Billerica, Massachusetts, USA) was used, following the manufacturer's protocol. The plate was read using a Bio-Plex 200 system (Bio Rad, Hertfordshire, United Kingdom). Thirteen cytokines and chemokines were assessed: Interferon (IFN)- $\gamma$, Interleukin (IL)- $1 \alpha$, IL-1 $\beta$, IL-1RA, IL-2, IL-4, IL-6, IL8, IL-10, IL-12p40, IL-12p70, granulocyte macrophage colonystimulating factor (GM-CSF), and tumor necrosis factor (TNF)$\alpha(12)$.

\section{Flow Cytometry}

Samples of perfusate $(4 \mathrm{ml})$ were collected into EDTA vacutainers, $0.4 \mathrm{ml}$ dimethyl sulfoxide (DMSO) was added and well-mixed; $2 \mathrm{ml}$ of this solution was then transferred into a cryogenic storage vial, moved to a CoolCell ${ }^{\circledR}$ Cell Freezing Container and stored in a $-80^{\circ} \mathrm{C}$ freezer. Immunophenotyping of the human perfusate samples was performed on a BD LSR II flow cytometer (Becton Dickinson, Oxford, United Kingdom). Leukocytes were identified and gated 
as CD45+ and their viability assessed using an eFluor ${ }^{\mathrm{TM}} 506$ viability dye (eBioscience, California, USA). Following this, a panel of antibodies was utilized to characterize $\mathrm{T}$ helper cells $(\mathrm{CD} 3 \varepsilon+\mathrm{CD} 4 \alpha+)$, cytotoxic $\mathrm{T}$ cells $(\mathrm{CD} 3 \varepsilon+\mathrm{CD} 8 \alpha+)$, doublepositive T cells (CD $3 \varepsilon+\mathrm{CD} 4 \alpha+\mathrm{CD} 8 \alpha+)$, double-negative T cells $(\mathrm{CD} 3 \varepsilon+\mathrm{CD} 4 \alpha-\mathrm{CD} 8 \alpha-), \gamma \delta \mathrm{T}$ cells $(\gamma \delta+), \mathrm{B}$ cells $(\mathrm{CD} 3 \varepsilon-\mathrm{CD} 21+)$, classical monocytes (CD14+CD163-), non-classical monocytes $(\mathrm{CD} 14+\mathrm{CD} 163+)$, immature neutrophils $(6 \mathrm{D} 10+2 \mathrm{~B} 2-)$, mature neutrophils $(6 \mathrm{D} 10+2 \mathrm{~B} 2+)$, mature eosinophils/basophils (6D10-2B2+), and natural killer cells (CD335+). Cells were treated with red blood cell lysing solution (BD Biosciences, United Kingdom), washed, and resuspended in $0.3 \mathrm{ml}$ of staining buffer. A $20 \mathrm{ml}$ quantity of e123count beads (eBioscience, California, USA) was added and samples were analyzed for $3 \mathrm{~min}$. All gating strategies and analysis were performed using FlowJo version 10.0.6 (12).

\section{Data Analysis}

The statistical testing was done with Graph Pad Prism 7 and IBM $^{\circledR}$ SPSS $^{\circledR}$ Statistics Version 25. A $p$-value of $<0.05$ was considered as statistically significant. Biomarker, Luminex ${ }^{\circledR}$ and flow results, donor and perfusion factors were analyzed using parametric and non-parametric tests, including Spearman rank correlation. The Bonferroni method was applied to correct for multiple testing in the correlation analyses.

\section{RESULTS}

Twelve discarded human kidneys, seven from donors after brain death (DBD) and five from donors after circulatory death (DCD), were perfused for a median (min-max) of 12.8 (6.1-24.1) h. Volume management was facilitated by replacement of the urine (UR) with Ringer's lactate in four (4/12, 33.3\%) NMP kidneys, and urine recirculation (URC) was applied in eight $(8 / 12,66.7 \%)$ kidney perfusions.

Table 1A illustrates the demographics for perfused DBD and DCD kidneys including donor risk indices. The median (minmax) UK kidney donor risk index [UKKDRI, (16)] was 1.9 (1.1-2.87); only three kidneys had a UKKDRI $<1.35$ (Table 1). Median (min-max) CIT was 20.5 (12.7-46.9) h, median (minmax) WIT was 12 (9-15) min for DCD kidneys. DBD kidneys experienced a shorter median (IQR) CIT with 17.5 (5.9) h compared to 22 (24.1) h in DCD kidneys, $p=0.05$. Median (min$\max )$ donor urine output prior to retrieval was $60(10-350) \mathrm{ml} / \mathrm{h}$. Median (min-max) donor serum creatinine and eGFR at the time point of retrieval were $64.5(32-208) \mu \mathrm{mol} / \mathrm{l}$ and 81 (29-247) $\mathrm{ml} / \mathrm{min} / 1.73 \mathrm{~m}^{2}$.

The median (min-max, IQR) duration of NMP was 12.8 (6.1$24.1,17.2)$ h. The median (min-max) hourly urine output during NMP was $54.4(1.7-471.9) \mathrm{ml} / \mathrm{h}$ and the median arterial flow was $370.8(100-787) \mathrm{ml} / \mathrm{min}$. The median (min-max) perfusate lactate during NMP was $12.8(4.4-20) \mathrm{mmol} / \mathrm{l}$ and the median (min-max) perfusate $\mathrm{pH}$ throughout the preservation period was 7.36 (7.16-7.62). A significantly longer NMP period could be achieved in kidneys with URC ( $n=3$ URC kidneys $12 \mathrm{~h}, n=1$ URC kidney $18 \mathrm{~h}, n=4$ URC kidneys $24 \mathrm{~h}$ ); median (IQR) NMP time of 21 (11.4) hours with URC vs. 7.2 (3) hours with UR $(n=$
2 UR kidneys $6 \mathrm{~h}, n=1$ UR kidney $8 \mathrm{~h}, n=1$ UR kidney $9 \mathrm{~h}$ ), $p=0.01$.

To compare similar adequate time points between URC and UR kidneys, the time interval for perfusate analyses was the start of NMP until hour 6 of NMP as all of the perfused kidneys reached at least $6 \mathrm{~h}$ of NMP. Table $1 \mathrm{~B}$ summarizes the hemodynamic and metabolic function parameters for the individual kidneys. A total of 135 perfusate samples were analyzed; 45 per assessment of NGAL/KIM-1/L-FABP, cytokines, and cells.

Table 2 shows the first and the 6-h measurements, as well as the $\triangle$ values for NGAL, KIM-1, and L-FABP in absolute numbers. Table $\mathbf{3 A}$ displays the flow cytometry analyses results for all $(n=12)$ perfused NMP kidneys for time points $30 \mathrm{~min}$, hours 1 and 6 after NMP-start. There were significantly more T-cells, CD4+ and NKT-cells in the perfusate after $6 \mathrm{~h}$ of NMP compared to $30 \mathrm{~min}$ after NMP-start. The content of monocytes, the intermediate type, was also significantly higher at hour 6 compared to the early measurements after initiation of NMP (Table 3A). Perfusate volume in NMP-kidneys was either managed with URC $(n=8)$ or UR $(n=4)$; we compared the potential efflux of cells between these two groups, shown in Table 3B. In regards to cell type and number of cells in the perfusate, there were no significant differences detectable between URC and UR kidneys throughout $6 \mathrm{~h}$ of NMP. Supplementary Table 1A displays cells in the perfusate of URC kidneys up to $24 \mathrm{~h}$. To visualize changes of the cell count in the perfusate over time, a heatmap comprising all 12 NMP kidneys is pictured in Figure 1A; the changes of the number of cells in perfusates of kidneys undergoing different volume management (URC or UR) are depicted in Figure 1B.

Table 4A shows the cytokine concentration measured in the perfusate after 1 and $6 \mathrm{~h}$ of NMP in the overall investigated cohort of 12 kidneys. Interleukin 8 was the only cytokine which increased significantly over time; $p=0.003,95 \%$ CI of difference $-21,204$ to $-2,633$. Table $4 \mathrm{~B}$ gives an overview of the potential effect of the type of perfusate volume management, URC or UR, on the concentration of cytokines in the perfusate. In both settings, URC and UR, IL- $1 ß$ decreased over time. There were no significant differences of $\Delta$ IL- $1 \beta$ in perfusates of URC or UR kidneys between NMP-start and hour 6, $p=0.09$. Supplementary Table $1 \mathrm{~B}$ shows cytokines in the perfusate of URC kidneys up to $24 \mathrm{~h}$. For visualization purposes, heatmaps for the cytokine concentration changes over time, were configured and are shown in Figures 2A,B. Figure 2A displays the development of cytokine changes over time in the overall cohort of 12 NMP kidneys, Figure 2B illustrates the stratification for URC and UR kidneys.

\section{Donor Factors in Context With Perfusate Measurements}

Perfusates of DBD kidneys had a significant lower number of leukocytes (median, IQR) after $6 \mathrm{~h}$ of NMP compared to DCD kidneys; $99,862(178,223)$ in DBD vs. 466,163 $(239,703)$ in DCD, $p=0.02$. There were less $\mathrm{T}$ cells (median, IQR), CD4+ and CD8+ T cells, in the perfusates 
TABLE 1A | Organ donor characteristics, procurement parameters, and reasons for discard of kidneys.

\begin{tabular}{|c|c|c|c|c|c|c|c|c|c|c|c|}
\hline & $\begin{array}{l}\text { Age in } \\
\text { years }\end{array}$ & Sex & $\mathrm{BMI}$ in $\mathrm{kg} / \mathrm{m}^{2}$ & Donor type & $\begin{array}{c}\text { Serum } \\
\text { creatinine } \\
{ }^{\circ} \text { retrieval in } \\
\mu \mathrm{mol} / \mathrm{L}\end{array}$ & $\begin{array}{c}\text { eGFR } \\
\text { @ retrieval in } \\
\mathrm{ml} / \mathrm{min} / 1.73 \\
\mathrm{~m}^{2}\end{array}$ & $\begin{array}{c}\text { WIT in } \\
\text { minutes }\end{array}$ & $\begin{array}{c}\text { CIT in hours }+ \\
\text { minutes }\end{array}$ & Hypertension & UKKDRI/KDRI & Reason for discard \\
\hline Kidney 1 & 59 & Male & 35.1 & DCD & 32 & 247 & 15 & $21+16$ & Yes & $1.10 / 1.21$ & Arteriosclerosis \\
\hline Kidney 2 & 60 & Male & 35.1 & DCD & 114 & 71 & 14 & $42+17$ & Yes & $1.50 / 1.60$ & Poor perfusion \\
\hline Kidney 3 & 44 & Male & 41.4 & $\mathrm{DCD}$ & 63 & 120 & 12 & $46+59$ & Yes & $1.22 / 1.13$ & Biopsy findings \\
\hline Kidney 4 & 66 & Female & 31.2 & DBD & 208 & 41 & n.a. & $15+9$ & No & $1.96 / 1 / 49$ & Patchy perfusion \\
\hline Kidney 5 & 70 & Female & 24.3 & DBD & 44 & 119 & n.a. & $17+30$ & Yes & $2.02 / 1.83$ & Stenosis of renal artery \\
\hline Kidney 6 & 74 & Female & 24.8 & DCD & 57 & 90 & 11 & 22 & Yes & $2.07 / 2.17$ & $\begin{array}{l}\text { Lesion on partner } \\
\text { kidney (monomorphic } \\
\text { cell infiltration) }\end{array}$ \\
\hline Kidney 7 & 71 & Female & 28.1 & DBD & 86 & 56 & n.a. & $46+47$ & Yes & $1.85 / 1.98$ & Anatomy, long CIT \\
\hline Kidney 8 & 78 & Female & 25.4 & DBD & 79 & 61 & n.a. & $18+22$ & Yes & $2.87 / 2.38$ & Vascular damage \\
\hline Kidney 9 & 71 & Female & 29.1 & DBD & 66 & 77 & n.a. & $21+4$ & No & $2.02 / 1.67$ & Organ size \\
\hline Kidney 10 & 47 & Female & 39.1 & DBD & 152 & 29 & n.a. & $12+41$ & No & $1.21 / 1.09$ & $\begin{array}{l}\text { Vascular damage, } \\
\text { patchy perfusion }\end{array}$ \\
\hline Kidney 11 & 62 & Female & 23.5 & DCD & 62 & 85 & 9 & $19+52$ & No & $1.67 / 1.50$ & Suspicion of cancer \\
\hline Kidney 12 & 76 & Female & 24.5 & DBD & 36 & 152 & n.a. & $15+26$ & Yes & $1.97 / 1.28$ & Long CIT \\
\hline
\end{tabular}

BMI, body mass index; $D C D$, donation after circulatory death; DBD, donation after brain death; eGFR, estimated glomerular filtration rate MDRD (modification of diet in renal disease); WIT, warm ischemia time; CIT, cold ischemia time. UKKDRI (15): Watson et al. (16); KDRI, OPTN KDRI/KDPI calculator. 
TABLE 1B | Hemodynamic and metabolic function parameters.

\begin{tabular}{|c|c|c|c|c|c|c|}
\hline & Kidney 1 & Kidney 2 & Kidney 3 & Kidney 4 & Kidney 5 & Kidney 6 \\
\hline Arterial pressure in $\mathrm{mmHg}($ mean, SD)* & $84.6 \pm 1.1$ & $82.5 \pm 7.8$ & $91.3 \pm 6.4$ & $90.3 \pm 1.3$ & $90.4 \pm 2.1$ & $88.9 \pm 1.7$ \\
\hline Arterial flow in $\mathrm{ml} / \mathrm{min}$ (mean, SD)* & $650 \pm 191.6$ & $294.5 \pm 74$ & $325 \pm 127.7$ & $271 \pm 54.6$ & $383.9 \pm 88.3$ & $474.1 \pm 149.7$ \\
\hline IRR in $\mathrm{ml} / \mathrm{min} / \mathrm{mmHg}$ (mean, SD) & $0.15 \pm 0.08$ & $0.3 \pm 0.09$ & $0.39 \pm 0.36$ & $0.36 \pm 0.18$ & $0.25 \pm 0.08$ & $0.2 \pm 0.09$ \\
\hline $\mathrm{pH}($ mean, SD)* & $7.39 \pm 0.12$ & $7.21 \pm 0.1$ & $7.24 \pm 0.17$ & $7.33 \pm 0.06$ & $7.4 \pm 0.04$ & $7.66 \pm 0.2$ \\
\hline Arterial $\mathrm{pO}_{2}$ in $\mathrm{kPa}(\text { mean, } \mathrm{SD})^{\star}$ & $15 \pm 1.6$ & $14.7 \pm 0.7$ & $12.6 \pm 2$ & $12 \pm 1.5$ & $13.8 \pm 0.6$ & $15 \pm 3$ \\
\hline Venous $\mathrm{pO}_{2}$ in $\mathrm{kPa}(\text { mean, } \mathrm{SD})^{\star}$ & $7.7 \pm 1.9$ & $7.6 \pm 0.8$ & $6.4 \pm 1.1$ & $7 \pm 2.4$ & $8 \pm 0.9$ & $7.4 \pm 0.9$ \\
\hline Arterial $\mathrm{pCO}_{2}$ in $\mathrm{kPa}(\text { mean, } \mathrm{SD})^{\star}$ & $4.3 \pm 0.9$ & $5.1 \pm 0.4$ & $5.9 \pm 0.7$ & $4.6 \pm 0.7$ & $5.1 \pm 0.9$ & $4.6 \pm 0.6$ \\
\hline Lactate level in $\mathrm{mmol} / /\left(\right.$ mean, SD) ${ }^{\star}$ & $13.4 \pm 1.62$ & $13.27 \pm 1.32$ & $12.19 \pm 3$ & $18.52 \pm 2.16$ & $5.65 \pm 3.8$ & $9.62 \pm 4$ \\
\hline Total glucose given in gram ${ }^{\star \star}$ & 2.4 & 3 & 1.2 & 0.75 & 3.2 & 0.55 \\
\hline Total urine output in ml & 828 & 286 & 10 & 105 & 1,285 & 445 \\
\hline Total urine output in $\mathrm{ml} /$ hour & 63.7 & 15.9 & 1.7 & 8.8 & 53.5 & 74.2 \\
\hline Urine recirculation yes/no & Yes & Yes & Yes & Yes & Yes & No \\
\hline \multirow[t]{2}{*}{ Time on the device (hours + min) } & $13+1$ & $18+3$ & $6+20$ & $12+35$ & $24+5$ & $6+10$ \\
\hline & Kidney 7 & Kidney 8 & Kidney 9 & Kidney 10 & Kidney 11 & Kidney 12 \\
\hline Arterial pressure in mmHg (mean, SD)* & $92.4 \pm 2.9$ & $92.2 \pm 2.8$ & $89.8 \pm 0.5$ & $91.64 \pm 2.3$ & $89.2 \pm 2.2$ & $90.3 \pm 2.1$ \\
\hline Arterial flow in $\mathrm{ml} / \mathrm{min}($ mean, SD) & $148.2 \pm 46.3$ & $123.5 \pm 79.16$ & $339.6 \pm 83.4$ & $240.7 \pm 120.9$ & $468.8 \pm 82.8$ & $664.9 \pm 228.1$ \\
\hline IRR in ml/min/mmHg (mean, SD)* & $0.76 \pm 0.5$ & $1.2 \pm 1.02$ & $0.3 \pm 0.12$ & $0.5 \pm 0.3$ & $0.19 \pm 0.05$ & $0.17 \pm 0.13$ \\
\hline $\mathrm{pH}($ mean, SD)* & $7.18 \pm 0.08$ & $7.2 \pm 0.1$ & $7.6 \pm 0.2$ & $7.33 \pm 0.1$ & $7.39 \pm 0.04$ & $7.43 \pm 0.02$ \\
\hline Arterial $\mathrm{pO}_{2}$ in $\mathrm{kPa}(\text { mean, } \mathrm{SD})^{\star}$ & $13.6 \pm 1.1$ & $14.6 \pm 2$ & $14 \pm 3.2$ & $13.5 \pm 2.9$ & $15.4 \pm 3.6$ & $12.45 \pm 0.8$ \\
\hline Venous $\mathrm{pO}_{2}$ in $\mathrm{kPa}$ (mean, SD)* & $8.3 \pm 1.5$ & $7.8 \pm 1.8$ & $6.4 \pm 1.3$ & $6.7 \pm 1.3$ & $7.1 \pm 1.6$ & $7.6 \pm 1.3$ \\
\hline Arterial $\mathrm{pCO}_{2}$ in $\mathrm{kPa}$ (mean, SD)* & $5.4 \pm 0.9$ & $4.8 \pm 1.1$ & $5 \pm 0.7$ & $4.5 \pm 0.9$ & $4.7 \pm 0.6$ & $4.6 \pm 0.4$ \\
\hline Lactate level in $\mathrm{mmol} / /(\text { mean, } \mathrm{SD})^{\star}$ & $16.18 \pm 2.14$ & $14.28 \pm 4.5$ & $16.34 \pm 2.9$ & $18.84 \pm 2.2$ & $9.19 \pm 2$ & $7.04 \pm 1.46$ \\
\hline Total glucose given in gram ${ }^{\star *}$ & 1 & 1.5 & 0.5 & 5 & 3 & 4.55 \\
\hline Total urine output in ml & 50 & 920 & 675 & 11,325 & 1,325 & 1,223 \\
\hline Total urine output in $\mathrm{ml} /$ hour & 8.3 & 102.2 & 84.4 & 471.9 & 55.2 & 51 \\
\hline Urine recirculation yes/no & No & No & No & Yes & Yes & Yes \\
\hline Time on the device (hours + min) & $6+5$ & $9+25$ & $8+10$ & $24+5$ & 24 & 24 \\
\hline
\end{tabular}

${ }^{*}$ Time-averaged longitudinal mean value compiled from hourly measurements over the course of the perfusion.

Circulating perfusate volume of $500 \mathrm{ml}$.

Parts of donor and perfusion characteristics of kidneys 6,8 and 9 without urine recirculation have been published previously (13). 
TABLE 2 | Observed perfusate biomarker concentrations.

\begin{tabular}{|c|c|c|c|c|}
\hline $\mathrm{NGAL}$ in $\mathrm{ng} / \mathrm{mL}$ with urine recirculation & First time point ${ }^{\star}$ & Last time point ${ }^{\star *}$ & Delta & Median (IQR) $)^{\star \star * *}$ \\
\hline Kidney 1 & 28.3 & 37.2 & 8.9 & $37.2(9.6)$ \\
\hline Kidney 2 & 67.1 & 102.1 & 35 & $97.9(32.1)$ \\
\hline Kidney 3 & 19.1 & 43.1 & 24 & $31(24)$ \\
\hline Kidney 4 & 60.2 & 54.3 & -5.9 & $59.8(6)$ \\
\hline Kidney 5 & 8.9 & 7.7 & -1.2 & $12(24.7)$ \\
\hline Kidney 11 & 10.4 & 32.9 & 22.5 & $36.8(34.1)$ \\
\hline Kidney 12 & 10.7 & 1.6 & -9.1 & $16.1(47.7)$ \\
\hline \multicolumn{5}{|l|}{ NGAL in $\mathrm{ng} / \mathrm{mL}$ without urine recirculation } \\
\hline Kidney 6 & 17.3 & 7.4 & -9.9 & $7.4(10.1)$ \\
\hline Kidney 7 & 100.9 & 117.2 & 16.3 & $103.5(16.3)$ \\
\hline Kidney 1 & 348.5 & 518.1 & 169.7 & $518.1(389.3)$ \\
\hline Kidney 2 & $1,132.7$ & $1,206.7$ & 74.1 & $1,213(77)$ \\
\hline Kidney 3 & 397.6 & 932.1 & 552.5 & $655.8(552.5)$ \\
\hline Kidney 4 & 731.1 & $1,144.3$ & 413.2 & $1,144(438.9)$ \\
\hline Kidney 5 & 170.5 & 429.8 & 259.4 & $724.2(618.8)$ \\
\hline Kidney 10 & 211.8 & 267.1 & 55.3 & $305.7(168)$ \\
\hline Kidney 11 & 73.9 & 436.5 & 362.6 & $518.2(489.4)$ \\
\hline Kidney 12 & 55.6 & 545.6 & 490 & $828.3(892.4)$ \\
\hline \multicolumn{5}{|l|}{$\mathrm{KIM}-1$ in $\mathrm{pg} / \mathrm{mL}$ without urine recirculation } \\
\hline Kidney 6 & 75.3 & 151.5 & 76.3 & $151.5(99.5)$ \\
\hline Kidney 3 & 31.1 & 0.7 & -30.4 & $15.9(30.4)$ \\
\hline Kidney 4 & 862.8 & 877.3 & 14.5 & $862.8(21.7)$ \\
\hline Kidney 5 & $<$ detection limit ${ }^{\star \star \star}$ & 13.6 & 13.6 & $26.9(39.7)$ \\
\hline Kidney 10 & 70.2 & 41.8 & -28.4 & $56(20.1)$ \\
\hline Kidney 11 & 88.4 & 138 & 49.6 & $138(36.6)$ \\
\hline Kidney 12 & 154.8 & $<$ detection limit ${ }^{\star \star \star}$ & -154.8 & $10.4(111.6)$ \\
\hline \multicolumn{5}{|c|}{ L-FABP in $\mathrm{ng} / \mathrm{mL}$ without urine recirculation } \\
\hline Kidney 6 & 289.2 & 369.5 & 80.3 & $357.2(79.7)$ \\
\hline Kidney 7 & 852.5 & 859.4 & 6.9 & $855.2(6.9)$ \\
\hline Kidney 8 & 1.8 & 27.3 & 25.5 & $22.4(25.5)$ \\
\hline Kidney 9 & 39.6 & $<$ detection limit ${ }^{\star \star \star}$ & -39.6 & $4.2(39.6)$ \\
\hline
\end{tabular}

${ }^{*} 1 \mathrm{~h}$ after perfusion start.

${ }^{* *} 6 h$ after perfusion start.

${ }^{* * *}$ Minimum detectable dose for assay $<6.25 \mathrm{ng} / \mathrm{ml}$ (L-FABP).

${ }^{* * * *}$ Time-averaged longitudinal value compiled from all measurements over the course of the perfusion; perfusate volume $=500 \mathrm{ml}$.

Some NGAL, KIM-1 and L-FABP measurements of kidneys 6, 8, and 9 without urine recirculation have been published previously (13). 
TABLE 3A | Flow cytometry results ${ }^{*}$ of $n=12$ NMP kidneys.

\begin{tabular}{|c|c|c|c|c|c|}
\hline & $30 \mathrm{~min}^{\star *}$ & $1 h^{\star *}$ & $6 h^{\star *}$ & $p$-value ${ }^{\star \star *}$ & $p$-value ${ }^{\star \star \star *}$ \\
\hline Total leukocytes & $166,023,158,326$ & $259,628,190,892$ & $268,659,355,959$ & 0.10 & 0.15 \\
\hline Total T Cells & $33,434,30,009$ & $53,971,35,611$ & $58,032,74,672$ & 0.14 & 0.03 \\
\hline CD4T cells & $11,716,20,791$ & $18,821,18,157$ & $24,095,49,548$ & 0.28 & 0.03 \\
\hline CD8T cells & $15,124,15,919$ & $27,610,26,454$ & $27,612,25,313$ & 0.13 & 0.10 \\
\hline NK T cells & $4,332,5,681$ & $4,997,4,677$ & 7,932, 8,762 & 0.25 & 0.03 \\
\hline Classical & $7,480,6,085$ & $18,017,19,586$ & $5,547,10,488$ & 0.04 & 0.96 \\
\hline Intermediate & $4,099,4,603$ & $11,412,11,400$ & $1,363,1,696$ & 0.01 & 0.03 \\
\hline Non-classical & $2,452,2,423$ & $5,341,6,669$ & $2,320,4,276$ & 0.01 & 0.97 \\
\hline Eosinophils & 393,786 & 439,742 & $711,1,473$ & 0.68 & 0.23 \\
\hline Neutrophils & 157,249 & 359,217 & $242,1,263$ & 0.84 & 0.99 \\
\hline
\end{tabular}

${ }^{\star}$ Number of cells in cells $/ \mathrm{ml}$; overall perfusate volume $=500 \mathrm{ml}$.

${ }^{* *}$ Time after start of NMP, values in median and IQR (interquartile range).

${ }^{* * *}$ Comparison $30 \mathrm{~min}$ with $1 \mathrm{~h}$ values.

${ }^{* * * *}$ Comparison 30 min with $6 \mathrm{~h}$ values.

TABLE 3B | Flow cytometry results* stratified for urine recirculation and urine replacement.

\begin{tabular}{|c|c|c|c|c|c|c|c|c|c|}
\hline & \multicolumn{3}{|c|}{ Kidneys with urine recirculation $(n=8)$} & \multicolumn{3}{|c|}{ Kidneys without urine recirculation $(n=4)$} & \multirow{2}{*}{$\begin{array}{c}p \text {-value }{ }^{\star \star \star} \\
30 \mathrm{~min}\end{array}$} & \multirow{2}{*}{$\begin{array}{c}p \text {-value } \\
1 \mathrm{~h}\end{array}$} & \multirow{2}{*}{$\begin{array}{c}p \text {-value } \\
6 \mathrm{~h}\end{array}$} \\
\hline & $30 \min ^{\star *}$ & $1 h^{\star \star}$ & $6 h^{\star *}$ & $30 \mathrm{~min}^{\star \star}$ & $1 h^{\star *}$ & $6 h^{\star \star}$ & & & \\
\hline Total leukocytes & $194,687,169,462$ & $301,435,173,632$ & $285,198,314,757$ & $112,250,125,008$ & $173,140,224,651$ & $131,250,371,186$ & 0.37 & 0.21 & 0.68 \\
\hline Total T Cells & $39,778,42,889$ & $54,203,18,377$ & $79,875,79,263$ & $21,809,28,131$ & $26,523,47,966$ & $28,027,69,184$ & 0.28 & 0.15 & 0.28 \\
\hline CD4T cells & $14,579,24,059$ & $24,088,14,444$ & $30,468,54,969$ & $6,123,9,731$ & $8,402,11,934$ & $9,112,40,426$ & 0.28 & 0.07 & 0.57 \\
\hline CD8T cells & $16,913,13,757$ & $33,008,19,555$ & $31,977,21,867$ & $11,074,18,395$ & $13,174,32,824$ & $12,905,23,162$ & 0.15 & 0.15 & 0.11 \\
\hline NK T cells & $2,943,5,681$ & $4,595,3,800$ & $8,050,11,446$ & $6,254 \pm 3,241$ & $7,870,5,795$ & $7,932,5,671$ & 0.68 & 0.37 & 0.93 \\
\hline B cells & $17,692,29,619$ & $18,707,26,760$ & $31,089,43,484$ & $18,933,64,538$ & $16,183,60,774$ & $19,205,271,378$ & 0.68 & 0.99 & 0.93 \\
\hline \multicolumn{10}{|l|}{ Monocytes } \\
\hline Classical & $6,310,10,731$ & $18,017,19,859$ & $7,188,11,711$ & $7,480,2,978$ & $15,596,19,757$ & $5,547,5,020$ & 0.99 & 0.93 & 0.99 \\
\hline Intermediate & $3,462,5,310$ & $9,255,11,880$ & 1,363, 2,799 & $4,180,3,842$ & $13,508,13,273$ & $1,292,1,749$ & 0.81 & 0.46 & 0.93 \\
\hline Non-classical & $2,743,3,285$ & $7,667,6,711$ & $3,790,3,645$ & $1,810,3,074$ & $2,960,2,963$ & $1,114,1,364$ & 0.15 & 0.05 & 0.05 \\
\hline Eosinophils & $545,12,346$ & $613.4,5,528$ & $1,122,24,421$ & 332,764 & 296,853 & 711,653 & 0.57 & 0.28 & 0.81 \\
\hline Neutrophils & 157, 202 & $379.1,156.2$ & $210.2,351.4$ & 105, 392 & 296,438 & $1,039,2,564$ & 0.88 & 0.49 & 0.20 \\
\hline NK cells & $11,780,56,253$ & $34,918,63,636$ & $26,187,48,707$ & $10,316,12,633$ & $19,607,22,801$ & $3,549,5,731$ & 0.57 & 0.21 & 0.07 \\
\hline Macrophages & $3,073,9,088$ & $4,433,11,636$ & $3,709,4,719$ & $6,469,6,217$ & $21,035,42,213$ & $5,988,15,180$ & 0.68 & 0.21 & 0.46 \\
\hline
\end{tabular}

${ }^{\star}$ Number of cells in cells $/ \mathrm{ml}$; overall perfusate volume $=500 \mathrm{ml}$.

${ }^{* *}$ Time after start of NMP, values in median and IQR (interquartile range).

${ }^{* * *} P$-value result of comparison with and without urine recirculation.

of DBD kidneys after $6 \mathrm{~h}$ of NMP compared to DCD organs; $30,071(22,961)$ in DBD vs. $102,356(56,002)$ in DCD, $p=0.01$.

Donor hypertension did not correlate with any of the perfusate markers measured.

Estimated glomerular filtration rate (eGFR) of the kidney donors and their corresponding serum creatinine were related with changes of IFN- $\gamma$ and IL- 6 over $6 \mathrm{~h}$ NMP without reaching significance. Perfusate NGAL after $6 \mathrm{~h}$ of NMP was insignificantly higher in kidneys from a donor with higher serum creatinine at the time of retrieval.

Duration of CIT did not correlate with any of the biomarkers (NGAL, KIM-1, and L-FABP), neither with number of leukocytes nor with cytokines in the perfusate. The correlations coefficients (Spearman's rho) and associated $p$-values are shown in Table 5; $p<0.001$ considered significant according to Bonferroni correction. 
A

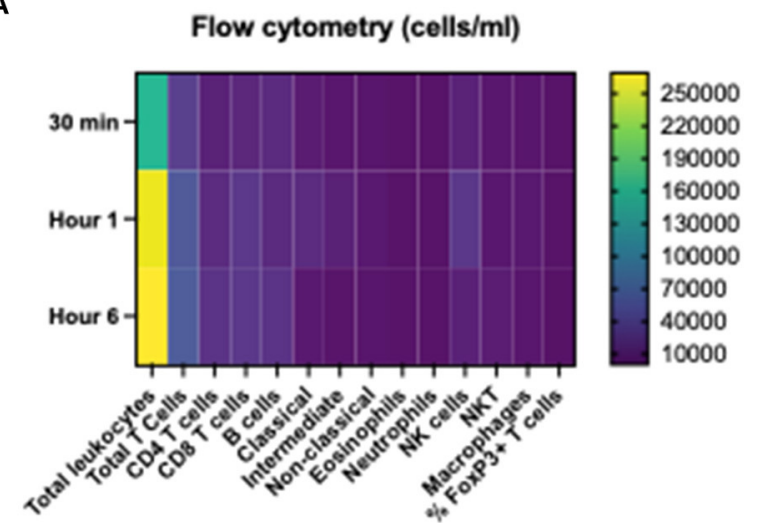

B

Flow cytometry (cells $/ \mathrm{ml}$ ) stratified for URC and UR

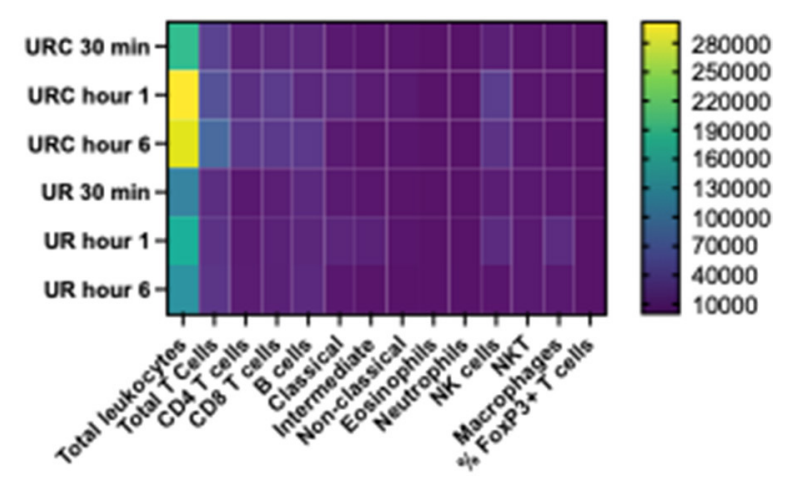

FIGURE 1 | (A) Heatmap visualizing results of flow cytometry analyses (cells/ml perfusate, 500 ml overall volume) of $n=12$ NMP kidneys; time points investigated: $30 \mathrm{~min}, 1$ and $6 \mathrm{~h}$ after NMP start. Median of cell count per measured entity per time point is displayed. (B) Heatmap visualizing results of flow cytometry analyses (cells/ml perfusate, $500 \mathrm{ml}$ overall volume) stratified for urine recirculation (URC) and urine replacement (UR) kidneys; time points investigated: $30 \mathrm{~min}$, 1 and $6 \mathrm{~h}$ after NMP start. Median of cell count per measured entity per time point is displayed.

TABLE 4A | Luminex results* of $n=12$ NMP kidneys.

\begin{tabular}{lccc}
\hline & $\mathbf{1} \mathbf{h}^{\star *}$ & $\mathbf{6} \mathbf{h}^{* *}$ & $\boldsymbol{p}$-value \\
\hline GM-CSF & $10,000,9,979$ & 27,208 & 0.77 \\
IFN- $\gamma$ & $10,000,0$ & $10,000,7,476$ & $>0.9$ \\
IL-10 & $2,159,9952$ & $1,743,3,646$ & $>0.9$ \\
IL-12p40 & $10,000,0$ & $10,000,7,481$ & $>0.9$ \\
IL-12p70 & $10,000,0$ & $10,000,0$ & $>0.9$ \\
IL-1RA & $453,1,845$ & $980,1,997$ & $>0.9$ \\
IL-1 $\alpha$ & $10,000,7,472$ & $10,000,7,387$ & $>0.9$ \\
IL-1 $\beta$ & $10,000,9,985$ & 14,164 & 0.94 \\
IL-2 & $10,000,7,497$ & $20,9,999$ & 0.94 \\
IL-4 & $10,000,0$ & $10,000,0$ & $>0.9$ \\
IL-6 & $45,9,007$ & $6,943,4,882$ & 0.89 \\
IL-8 & 24,872 & $17,625,196,167$ & 0.003 \\
TNF- $\alpha$ & 10,214 & 398,295 & $>0.9$ \\
\hline
\end{tabular}

*Number of cells in pg/ml; overall perfusate volume $=500 \mathrm{ml}$.

${ }^{* *}$ Time after start of NMP, values in median and IQR (interquartile range).

GM-CSF, granulocyte-macrophage colony-stimulating factor; IFN, interferon; IL, interleukin; TNF, tumor necrosis factor.

\section{NMP Perfusion Hemodynamics}

Renal arterial flow in $\mathrm{ml} / \mathrm{min}$ after $6 \mathrm{~h}$ of NMP correlated insignificantly with the CD8+ cell count. Overall, perfusates of kidneys with higher arterial flow had lower TNF- $\alpha$ levels. Kidneys from donors with higher eGFR at time of retrieval developed a significantly better arterial flow until hour 6 of NMP.

Figure $3 \mathrm{~A}$ displays a comparison of CIT and donor characteristics stratified for median (IQR) arterial flow, 303.5 (186) $\mathrm{ml} / \mathrm{min}$ at hour 6 after NMP start. There was no significant difference in duration of CIT $(p=0.7)$ and donor age $(p=0.8)$ for NMP kidneys reaching higher or lower arterial flow than the median of $303.5 \mathrm{ml} / \mathrm{min}$. Donor eGFR $(p=0.002)$ was higher and donor serum creatinine lower $(p=0.002)$ in NMP kidneys reaching a higher arterial flow than the median.

Arterial flow after $6 \mathrm{~h}$ of NMP and median arterial flow correlated inversely. Perfusate lactate measured at hour 6 after NMP start was significantly lower in NMP kidneys reaching an arterial flow higher than the median of $303.5 \mathrm{ml} / \mathrm{min} ; p=0.004$, shown in Figure 3B. The duration of NMP had no impact on reaching higher or lower median arterial flow; $p=0.6$, depicted in Figure 3B.

Perfusate $\mathrm{pH}$ measured at hour 6 after NMP-start correlated significantly with median $\mathrm{pH}$ over time. There was a trend toward a more physiological $\mathrm{pH}$ and lower NGAL levels of kidney perfusates with higher arterial flow after $6 \mathrm{~h}$ of NMP. Figure 3B displays the relation of NGAL, $\mathrm{pH}$ and lactate, all measured at hour 6, with arterial flow. Kidneys with arterial flows higher than the median of $303.5 \mathrm{ml} / \mathrm{min}$ had non-significantly lower NGALperfusate levels $(p=0.07)$ and a more physiological $\mathrm{pH}(p=0.06)$ but significantly lower perfusate lactate levels $(p=0.004)$.

NMP kidneys with higher arterial flow had non-significantly higher volume of hourly urine output; $p=0.7$, shown in Figure 3B. There was also a link between KIM-1 levels after $6 \mathrm{~h}$ of NMP and the change of KIM-1 in the perfusate within the first $6 \mathrm{~h}$ of NMP. The correlations coefficients (Spearman's rho) and associated $p$-values are shown in Table 5; $p<0.001$ considered significant according to Bonferroni correction.

\section{Volume Management}

The application of URC led to significantly longer periods of NMP; $p=0.01$, shown in Figure 3C. There were no other significant correlations of type of volume management, URC or UR, in regard to donor (type of donor, age, cause of death, serum creatinine, and eGFR), preservation (CIT, WIT), and hemodynamic factors (arterial flow during NMP). Perfusate lactate after $6 \mathrm{~h}$ of NMP was comparable between URC and UR kidneys; $p=0.6$, displayed in Figure 3C. 
TABLE 4B | Luminex results ${ }^{\star}$ stratified for urine recirculation and urine replacement.

\begin{tabular}{|c|c|c|c|c|c|c|}
\hline & \multicolumn{2}{|c|}{ Kidneys with urine recirculation $(n=8)$} & \multicolumn{2}{|c|}{ Kidneys without urine recirculation $(n=4)$} & \multirow[t]{2}{*}{$p$-value ${ }^{\star * *} 1 \mathrm{~h}$} & \multirow[t]{2}{*}{$p$-value ${ }^{* \star \star} 6 \mathrm{~h}$} \\
\hline & $1 h^{\star \star}$ & $6 h^{\star *}$ & $1 h^{\star \star}$ & $6 h^{* *}$ & & \\
\hline GM-CSF & $420.6,9,993$ & $53.5,2,300$ & 10,000, 0 & $12.6,27.7$ & 0.11 & 0.21 \\
\hline $\mathrm{IFN}-\gamma$ & 10,000, 0 & $10,000,9,982$ & $10,000,0$ & $10,000,0$ & $>0.9$ & 0.42 \\
\hline IL-10 & 2,160, 8196 & $1,890,3,727$ & $5,023,9,988$ & $1,214,3,607$ & $>0.9$ & $>0.9$ \\
\hline IL-12p40 & $10,000,0$ & $10,000,7,481$ & $10,000,0$ & $10,000,7,499$ & $>0.9$ & 0.83 \\
\hline IL-12p70 & $10,000,0$ & $10,000,0$ & $10,000,0$ & $10,000,7,493$ & $>0.9$ & $>0.9$ \\
\hline IL-1RA & $1,214,2,229$ & $1,933,2,710$ & $453.7,419.7$ & 192.6, 969 & 0.68 & 0.26 \\
\hline IL-1 $1 \alpha$ & $10,000,0$ & $10,000,9,935$ & $5,010,9,986$ & $10,000,0$ & 0.09 & 0.42 \\
\hline $\mathrm{IL}-1 \beta$ & $5,082,9,986$ & $113.7,7,534$ & $10,000,7,499$ & $1.2,3$ & 0.67 & 0.02 \\
\hline IL-2 & $10,000,9,998$ & $19.7,7,518$ & $10,000,0$ & $5,001,9,999$ & 0.42 & 0.53 \\
\hline IL-4 & $10,000,0$ & $10,000,0$ & $10,000,0$ & $10,000,0$ & $>0.9$ & $>0.9$ \\
\hline IL-6 & $4,493,9,040$ & $8,496,4,829$ & $19.6,47.7$ & $5,224,14,384$ & 0.11 & 0.68 \\
\hline IL-8 & $66.1,10,943$ & $16,501,35,438$ & $23.9,55.5$ & $17,625,9,744$ & 0.77 & 0.89 \\
\hline TNF- $\alpha$ & $18.4,560.8$ & $459.7,1,277$ & $8.9,9.4$ & $354.7,221.3$ & 0.46 & 0.37 \\
\hline
\end{tabular}

*Number of cells in pg/ml; overall perfusate volume $=500 \mathrm{ml}$.

${ }^{* *}$ Time after start of NMP, values in median and IQR (interquartile range).

${ }^{* * *} P$-value result of comparison with and without urine recirculation.

GM-CSF, granulocyte-macrophage colony-stimulating factor; IFN, interferon; IL, interleukin; TNF, tumor necrosis factor.

\section{A}

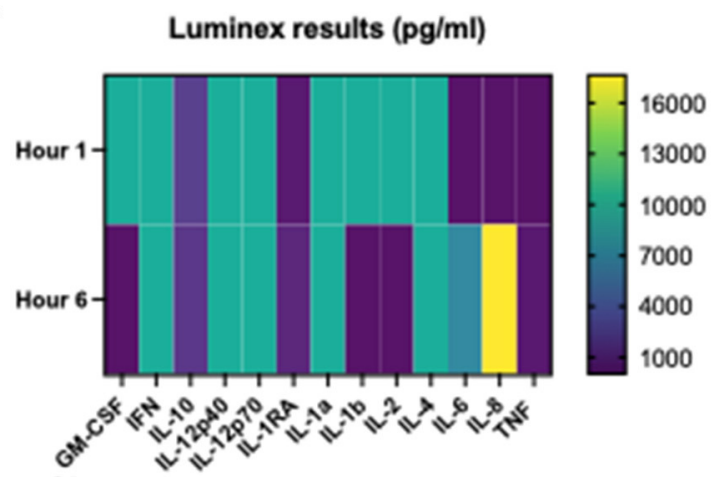

B

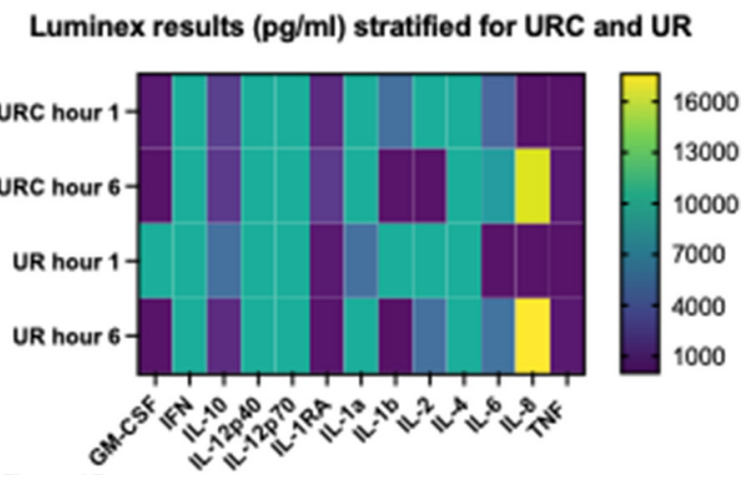

FIGURE 2 | (A) Heatmap visualizing Luminex results (pg/ml) of $n=12$ NMP kidneys; time points investigated: 1 and $6 \mathrm{~h}$ after NMP start. Median cytokine concentration per measured entity per time point is displayed. (B) Heatmap visualizing Luminex results (pg/ml) stratified for urine recirculation (URC) and urine replacement (UR) kidneys; time points investigated: 1 and $6 \mathrm{~h}$ after NMP start. Median cytokine concentration per measured entity per time point is displayed.

The correlation coefficients (Spearman's rho) and associated $p$-values are shown in Table 5; $p<0.001$ considered significant according to Bonferroni correction.

\section{Biomarkers of Renal Injury}

After application of the Bonferroni correction for multiple testing, there were no significant correlation of NGAL, KIM-1, and L-FABP with other parameters measured in the perfusate. There was a connection between NGAL levels after $6 \mathrm{~h}$ of NMP and the concentration of GM-CSF and its change over time, $\triangle \mathrm{GM}-\mathrm{CSF}$, in the perfusate. The concentration of IL$1 \beta$ was non significantly higher in perfusates with higher 6-h NGAL levels. Perfusates with higher KIM-1 levels after $6 \mathrm{~h}$ of NMP had a higher count of non-classical monocytes. Perfusates with higher L-FABP levels at hour 6 after NMP start had also non-significantly more macrophages in the perfusate. The correlations coefficients (Spearman's rho) and associated $p$-values are shown in Table 5; $p<0.001$ considered significant according to Bonferroni correction.

\section{DISCUSSION}

We herein report for the first time the results of measuring several biomarkers, including cytokines and leukocytes, in a normothermic human kidney perfusion model comparing a novel approach of urine recirculation (URC) to facilitate perfusate homeostasis and volume control $(12,13)$ with the technique of replacement of excreted urine using Ringer's lactate. Urine recirculation led to NMP durations up to $24 \mathrm{~h}$ and biomarker could be detected and analyses throughout these 
TABLE 5 | Correlation of hemodynamic and metabolic parameters with perfusate biomarker, cells, and cytokines.

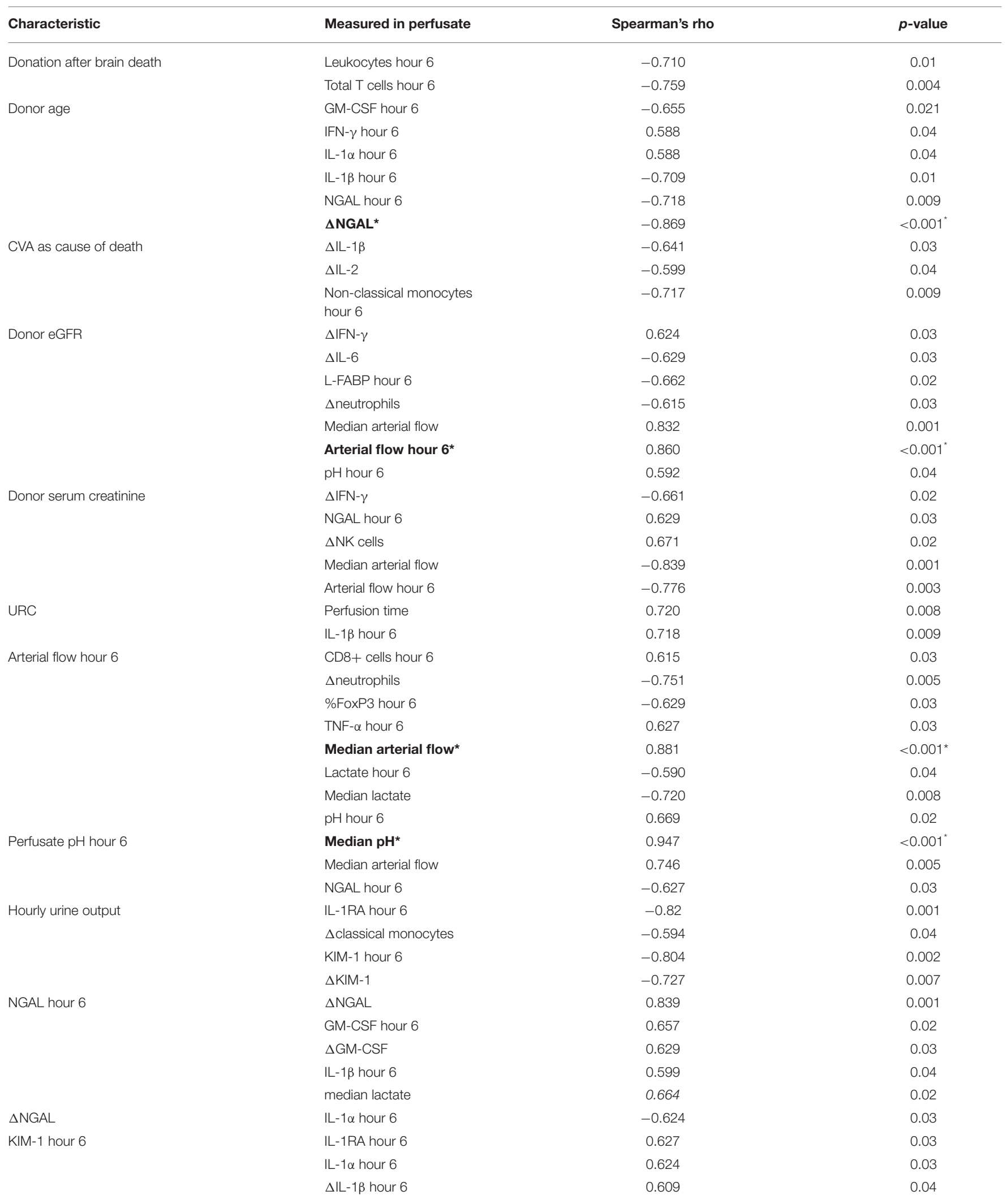


TABLE 5 | Continued

\begin{tabular}{|c|c|c|c|}
\hline Characteristic & Measured in perfusate & Spearman's rho & $p$-value \\
\hline & $\Delta$ intermediate monocytes & 0.594 & 0.04 \\
\hline & $\begin{array}{l}\Delta \text { non-classical } \\
\text { monocytes }\end{array}$ & 0.720 & 0.008 \\
\hline & $\Delta \mathrm{NK}$ cells & 0.608 & 0.04 \\
\hline \multirow[t]{2}{*}{ L-FABP hour 6} & IL-2 hour 6 & -0.599 & 0.04 \\
\hline & macrophages hour 6 & 0.641 & 0.03 \\
\hline \multirow[t]{3}{*}{$\Delta \mathrm{L}-\mathrm{FABP}$} & IL-2 hour 6 & -0.626 & 0.03 \\
\hline & $\Delta$ eosinophils & -0.650 & 0.02 \\
\hline & $\begin{array}{l}\Delta \text { non-classical } \\
\text { monocytes }\end{array}$ & -0.594 & 0.04 \\
\hline
\end{tabular}

$\Delta$, delta (difference start-6-h perfusion value); URC, urine recirculation; CVA, cerebrovascular accident; GM-CSF, granulocyte macrophage-colony stimulating factor; IL, interleukin; IFN, interferon; NK, natural killer; NGAL, neutrophil gelatinase-associated lipocalin; L-FABP, liver-type fatty acid-binding protein; KIM-1, kidney injury molecule 1; TNF, tumor necrosis factor.

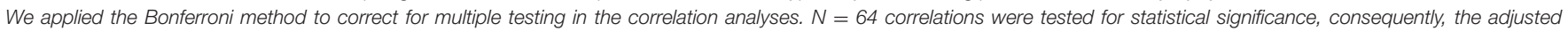
significance level is 0.05 divided by 64.

${ }^{\star} P$-values $<0.00078(<0.001)$ were considered statistically significant.

perfusions. The focus of this manuscript, however, was on the time point "hour 6" post perfusion start, as this was the latest comparable time point both, URC and UR NMP kidneys reached (17). Moreover, we focused on correlations with perfusion parameters and donor characteristics which are readily available at the time of decision making if a kidney is deemed transplantable after normothermic preservation and evaluation or declined for clinical use. This approach was chosen to examine some possible surrogate parameters, captured during perfusion, for organ viability. Our analyses were performed in a model of discarded human kidneys, therefore the important link to outcomes after successful transplantation is not available and no statements can be made in regard to estimating probable occurrence of delayed or primary non-function in the clinical setting.

The Cambridge group of Clatworthy, Ferdinand et al. (11), reported on NMP kidneys with higher inflammatory gene expression detectable in recipients who experienced prolonged DGF after receiving an NMP kidney. In their analyses, the course of $2 \mathrm{~h}$ NMP led to an upregulation of oxidative phosphorylation, but also an upregulation of a number of genes important for immune and inflammatory processes with NFkB induced TNF$\alpha$ signaling as the major part of it (11). We detected an increase of TNF- $\alpha$ in perfusates in URC and UR kidneys, but the change over time was insignificant and more importantly, there was no difference at any time of perfusate TNF- $\alpha$ between the URC and UR kidneys. However, interestingly renal arterial flow (in URC and UR kidneys) was higher in kidneys with lower perfusate TNF- $\alpha$ which implicates a link between inflammatory potential of the perfusate and one of the best-studied hemodynamic parameters, especially in a pressure-fixed system in which increasing arterial flow over time is a parameter of kidney function $(6,18)$. In concordance with Ferdinand et al. (11), we also saw an increase of IL- 8 in our perfusates over time. In line with IL-8, IL-6 increased over time in URC and UR kidneys without significant differences, but was not associated directly with any parameters identifying organ function during kidney NMP. However, both interleukins are known to be inflammatory with possible negative effects on renal parenchyma (19-21) and could be a future target for ex-situ organ treatment to prevent detrimental effects for the organ recipient. Our data revealed that IL-1 $\beta$ was higher in perfusates with higher content of NGAL and KIM-1 which are well-known markers for impaired kidney function and, NGAL at least, available to be measured in the clinical routine (2, 22-24). Another cytokine which could become of interest in future in a dynamic, normothermic preservation setting is GM-CSF. It is an immunregulatory cytokine which has been studied extensively recently due to its potential association with hyperinflammation in COVID-19 (25). GM-CSF is proinflammatory and plays a role in activation of macrophages and antigen-presenting cells (26). Whereas, in patients, as described in the literature, increased GM-CSF levels are associated with increased cytokines IL- 6 , TNF- $\alpha$, IFN- $\gamma$, and IL-18 (25, 27), in our perfusates GM-CSF decreased over time. In perfusates with higher NGAL, GM-CSF was also higher compared to perfusates with lower NGAL which could potentially indicate less organ damage.

The Cambridge group did not detect any differences between DBD and DCD organs in terms of inflammatory gene signature they described. The only difference we could observe between DBD and DCD organs was the significant higher number of leukocytes, CD4+ and CD8+ T-cells in DCD-perfusates after $6 \mathrm{~h}$ of NMP compared to DBD organs; independent of URC or UR. This finding could be representing the cessation of blood flow in DCD organs and warm ischemia time prior to the start of retrieval, as the donor leukocytes have still a "route out" in DBD kidneys. In future, it will be interesting to compare DBD kidneys not only with DCD ones, but also with kidneys procured after normothermic regional perfusion.

The most relevant findings of our analyses were the clear connection of perfusate lactate, perfusate $\mathrm{pH}$ and urine output with several kidney function parameters in the donor as well as 
A

Cold ischemia time and donor characteristics stratified for median arterial flow at hour 6 after perfusion start

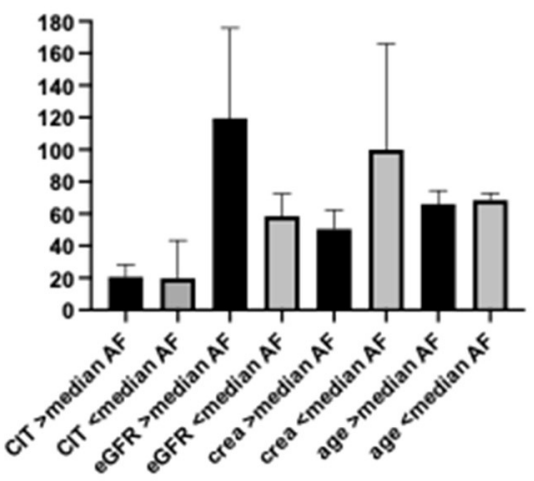

B Biomarker, hourly urine excretion and NMP duration stratified for
median arterial flow at hour 6 after perfusion start

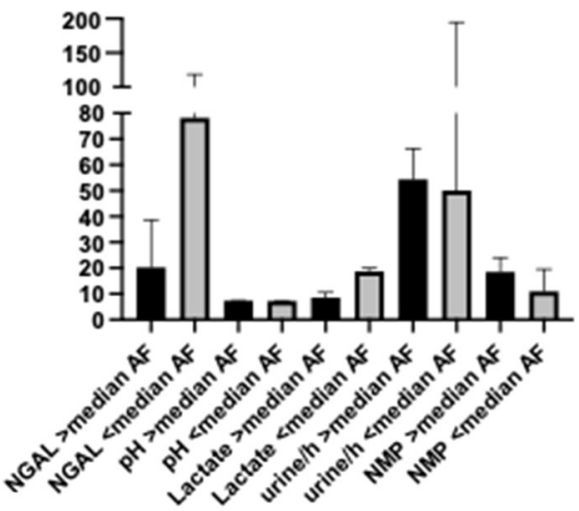

C

NMP duration, arterial flow and lactate stratified for urine and volume management

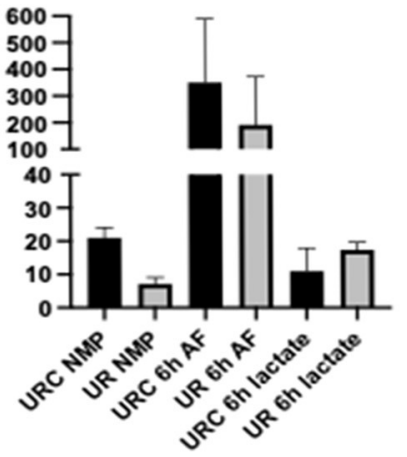

FIGURE 3 | (A) Comparison of cold ischemia time (CIT), estimated donor glomerular filtration rate (eGFR), donor serum creatinine (crea) at time point of retrieval, and donor age (age) stratified for median arterial flow (303.5 ml/min) after $6 \mathrm{~h}$ of NMP start; depicted values (column) of parameters are in median and IQR. (B) Comparison of perfusate NGAL, perfusate $\mathrm{pH}$, perfusate lactate at hour 6 after NMP start, hourly urine excretion, and NMP duration stratified for median arterial flow (303.5 ml/min) after $6 \mathrm{~h}$ of NMP start; depicted values (column) of parameters are in median and IQR. (C) Comparison of NMP duration, arterial flow at hour 6 after NMP start and perfusate lactate at hour 6 after NMP start stratified for perfusate volume management (urine recirculation URC of urine replacement UR); depicted values (column) of parameters are in median and IQR.

with published kidney injury markers as NGAL, KIM-1, and LFABP (2). Perfusate lactate was lower the higher the arterial flow was and also, potentially important for defining timing for exsitu organ assessment, the 6-h values of lactate, renal arterial flow and $\mathrm{pH}$ correlated with their respective median values. NGAL, a biomarker we would define as a routinely available biomarker (22), was also higher in perfusates with higher lactates and could be a surrogate biomarker for the donor kidney function on the circuit as it correlated significantly with donor creatinine and eGFR. Urine output during NMP, a marker implemented in the Hosgood and Nicholson score already, was associated positively with KIM-1 levels in the perfusate. In addition to our finding that biomarkers can be measured and correlated with transplant factors, overall NMP time itself did not correlate with any of the parameters measured. In particular, there was no association of a preservation period of $6 \mathrm{~h}$ and beyond with arterial flow and inflammatory potential of the perfusate. Such a finding could be crucial for implementing longer-term kidney NMP in the clinical routine.

Our results, gained from an ex-situ NMP setting cannot be correlated with clinical study results yet, but different cytokine and immune cell patterns do offer an important target to invest more research, particularly in forthcoming clinical use of the NMP device. The downside of performing cell and cytokine analyses solely in the perfusate and not in the tissue or from any other components in the circuit, is a limitation of our investigation, is the missing answer to the question of where the immune cells were possibly migrating to.

To summarize, clinically available perfusion parameters as perfusate lactate, $\mathrm{pH}$ and NGAL correlate well with donor 
characteristics, renal arterial flow, cytokines, immune cell changes, and KIM-1 in a discarded human NMP model. Potentially, lactate, $\mathrm{pH}$ and NGAL become a trinity to support decisions and fulfill the criteria to be diagnostic, predictive, and therapeutic biomarkers (28) in future for longer-term kidney NMP. In a non-transplant model long-term perfusion by applying URC was feasible and safe and also $6 \mathrm{~h}$ of NMP with UR seem to be applicable in a clinical transplant setting. Kidney NMP beyond 1 or $2 \mathrm{~h}$ might be helpful and instrumental in screening and discover markers indicating primary non-function of suboptimal organs (29). Therefore, these parameters should be considered as additional viability markers expanding the current decision-making score developed by Hosgood et al. (6).

\section{DATA AVAILABILITY STATEMENT}

The raw data supporting the conclusions of this article will be made available by the authors, without undue reservation.

\section{AUTHOR CONTRIBUTIONS}

AW designed the study, involved in the development of the perfusion device, performed the perfusions, collected and analysed the data, and wrote the manuscript. JS and JF performed the Luminex and FACS analyses. ML, JH, and RP were involved in data analyses, interpretation, and revision of the manuscript. CC and PF were instrumental for the study setup, the development of the perfusion device, and revision of the manuscript. All authors contributed to the article and approved the submitted version.

\section{FUNDING}

This research was supported by the Oxford Centre for Drug Delivery Devices under Programme Grant EP/L024012/1 from the UK's Engineering and Physical Sciences Research Council.

\section{SUPPLEMENTARY MATERIAL}

The Supplementary Material for this article can be found online at: https://www.frontiersin.org/articles/10.3389/fmed. 2021.801098/full\#supplementary-material

Supplementary Table 1A | Flow cytometry results for urine recirculation (URC) for $30 \mathrm{~min}, 1,6,12$, and $24 \mathrm{~h}$ after start of normothermic perfusion; 8 URC kidneys reached $12 \mathrm{~h}, 5 \mathrm{URC}$ kidneys $18 \mathrm{~h}$, and 4 URC kidneys $24 \mathrm{NMP}$ h.

Supplementary Table 1B | Luminex results for urine recirculation (URC) for 1, 6, 12 , and $24 \mathrm{~h}$ after start of normothermic perfusion; 8 URC kidneys reached $12 \mathrm{~h}, 5$ URC kidneys $18 \mathrm{~h}$, and 4 URC kidneys $24 \mathrm{NMP} \mathrm{h}$.

\section{REFERENCES}

1. Moers C, Smits JM, Maathuis M-HJ, Treckmann J, van Gelder F, Napieralski $\mathrm{BP}$, et al. Machine perfusion or cold storage in deceased-donor kidney transplantation. N Engl J Med. (2009) 360:7-19. doi: 10.1056/NEJMoa08 02289

2. Bonventre JV, Vaidya VS, Schmouder R, Feig P, Dieterle F. Next-generation biomarkers for detecting kidney toxicity. Nat Biotechnol. (2010) 28:43640. doi: 10.1038/nbt0510-436

3. Hosgood SA, Nicholson ML. First in man renal transplantation after ex vivo normothermic perfusion. Transplantation. (2011) 92:735-8. doi: 10.1097/TP.0b013e31822d4e04

4. Guzzi F, Knight SR, Ploeg RJ, Hunter JP. A systematic review to identify whether perfusate biomarkers produced during hypothermic machine perfusion can predict graft outcomes in kidney transplantation. Transpl Int. (2020) 33:590-602. doi: 10.1111/tri.13593

5. Parikh CR, Hall IE, Bhangoo RS, Ficek J, Abt PL, Thiessen-Philbrook H, et al. Associations of perfusate biomarkers and pump parameters with delayed graft function and deceased donor kidney allograft function. Am J Transplant Off J Am Soc Transplant Am Soc Transpl Surg. (2016) 16:152639. doi: 10.1111/ajt.13655

6. Hosgood SA, Thompson E, Moore T, Wilson $\mathrm{CH}$, Nicholson ML. Normothermic machine perfusion for the assessment and transplantation of declined human kidneys from donation after circulatory death donors. Br J Surg. (2018) 105:388-94. doi: 10.1002/bjs. 10733

7. Turner J-E, Becker M, Mittrücker H-W, Panzer U. Tissueresident lymphocytes in the kidney. J Am Soc Nephrol. (2018) 29:389-99. doi: 10.1681/ASN.2017060599

8. Munro DAD, Hughes J. The origins and functions of tissueresident macrophages in kidney development. Front Physiol. (2017) 8:837. doi: 10.3389/fphys.2017.00837

9. Stone JP, Ball AL, Critchley WR, Major T, Edge RJ, Amin K, et al. Ex vivo normothermic perfusion induces donor-derived leukocyte mobilization and removal prior to renal transplantation. Kidney Int Rep. (2016) 1:2309. doi: 10.1016/j.ekir.2016.07.009
10. Stone JP, Critchley WR, Major T, Rajan G, Risnes I, Scott H, et al. Altered immunogenicity of donor lungs via removal of passenger leukocytes using ex vivo lung perfusion. Am J Transplant Off J Am Soc Transplant Am Soc Transpl Surg. (2016) 16:33-43. doi: 10.1111/ajt.13446

11. Ferdinand JR, Hosgood SA, Moore T, Ferro A, Ward CJ, Castro-Dopico $\mathrm{T}$, et al. Cytokine absorption during human kidney perfusion reduces delayed graft function-associated inflammatory gene signature. Am J Transplant Off J Am Soc Transplant Am Soc Transpl Surg. (2020) 21:218899. doi: 10.1111/ajt.16371

12. Weissenbacher A. Normothermic Kidney Preservation. Oxford: University of Oxford (2018). Available online at: http://purl.org/dc/dcmitype/text; https:// ora.ox.ac.uk/objects/uuid:57ae08d0-bf5c-422d-af85-893e15e6ec7c (accessed June 7, 2020).

13. Weissenbacher A, Lo Faro L, Boubriak O, Soares MF, Roberts IS, Hunter JP, et al. Twenty-four-hour normothermic perfusion of discarded human kidneys with urine recirculation. Am J Transplant Off J Am Soc Transplant Am Soc Transpl Surg. (2018) 19:178-92. doi: 10.26226/morressier.5a38ffa3d462b8029238b4f6

14. Weissenbacher A, Voyce D, Ceresa CDL, Soares MF, Roberts IS, Hunter JP, et al. Urine recirculation improves hemodynamics and enhances function in normothermic kidney perfusion. Transplant Direct. (2020) 6:e541. doi: 10.1097/TXD.0000000000000985

15. NHSBT Policy POL186/10. Kidney Transplantation: Deceased Donor Allocation. (2019). Available online at: https://nhsbtdbe.blob.core.windows. net/umbraco-assets-corp/16915/kidney-allocation-policy-pol186.pdf

16. Watson CJE, Johnson RJ, Birch R, Collett D, Bradley JA. A simplified donor risk index for predicting outcome after deceased donor kidney transplantation. Transplantation. (2012) 93:314-8. doi: 10.1097/TP.0b013e31823f14d4

17. Weissenbacher A, Huang H, Surik T, Lo Faro ML, Ploeg RJ, Coussios $\mathrm{CC}$, et al. Urine recirculation prolongs normothermic kidney perfusion via more optimal metabolic homeostasis-a proteomics study. Am J Transplant Off J Am Soc Transplant Am Soc Transpl Surg. (2021) 21:174053. doi: 10.1111/ajt.16334

18. Rijkse E, de Jonge J, Kimenai HJaN, Hoogduijn MJ, de Bruin RWF, van den Hoogen MWF, et al. Safety and feasibility of $2 \mathrm{~h}$ of normothermic machine 
perfusion of donor kidneys in the Eurotransplant Senior Program. BJS Open. (2021) 5:zraa024. doi: 10.1093/bjsopen/zraa024

19. De Perrot M, Sekine Y, Fischer S, Waddell TK, McRae K, Liu M, et al. Interleukin-8 release during early reperfusion predicts graft function in human lung transplantation. Am J Respir Crit Care Med. (2002) 165:2115. doi: $10.1164 /$ ajrccm.165.2.2011151

20. Durlacher-Betzer K, Hassan A, Levi R, Axelrod J, Silver J, Naveh-Many T. Interleukin- 6 contributes to the increase in fibroblast growth factor 23 expression in acute and chronic kidney disease. Kidney Int. (2018) 94:31525. doi: 10.1016/j.kint.2018.02.026

21. Scheller J, Chalaris A, Schmidt-Arras D, Rose-John S. The pro- and antiinflammatory properties of the cytokine interleukin-6. Biochim Biophys Acta. (2011) 1813:878-88. doi: 10.1016/j.bbamcr.2011.01.034

22. Devarajan P. Review: neutrophil gelatinase-associated lipocalin: a troponinlike biomarker for human acute kidney injury. Nephrol Carlton Vic. (2010) 15:419-28. doi: 10.1111/j.1440-1797.2010.01317.x

23. Bonventre JV. Kidney injury molecule-1 (KIM-1): a urinary biomarker and much more. Nephrol Dial Transplant Off Publ Eur Dial Transpl Assoc Eur Ren Assoc. (2009) 24:3265-8. doi: 10.1093/ndt/gfp010

24. Han WK, Bailly V, Abichandani R, Thadhani R, Bonventre JV. Kidney Injury Molecule-1 (KIM-1): a novel biomarker for human renal proximal tubule injury. Kidney Int. (2002) 62:237-44. doi: 10.1046/j.1523-1755.2002.00433.x

25. Mehta P, Porter JC, Manson JJ, Isaacs JD, Openshaw PJM, McInnes IB, et al. Therapeutic blockade of granulocyte macrophage colony-stimulating factor in COVID-19-associated hyperinflammation: challenges and opportunities. Lancet Respir Med. (2020) 8:822-30. doi: 10.1016/S2213-2600(20)30267-8

26. Mehta HM, Malandra M, Corey SJ. G-CSF and GM-CSF in neutropenia. J Immunol Baltim Md. (2015) 195:1341-9. doi: 10.4049/jimmunol.1500861

27. Yoshihara S, Li Y, Xia J, Danzl N, Sykes M, Yang Y-G. Posttransplant hemophagocytic lymphohistiocytosis driven by myeloid cytokines and vicious cycles of T-cell and macrophage activation in humanized mice. Front Immunol. (2019) 10:186. doi: 10.3389/fimmu.2019. 00186
28. Carlomagno N, Incollingo P, Tammaro V, Peluso G, Rupealta N, Chiacchio G, et al. Diagnostic, predictive, prognostic, and therapeutic molecular biomarkers in third millennium: a breakthrough in gastric cancer. BioMed Res Int. (2017) 2017:7869802. doi: 10.1155/2017/7869802

29. Hosgood SA, Nicholson ML. A short period of normothermic machine perfusion may not be able to predict primary nonfunction in uncontrolled circulatory death kidneys. Transplantation. (2021) 105:e11-2. doi: 10.1097/TP.0000000000003415

Conflict of Interest: PF and CC are co-founders of OrganOx R Limited, receive consultancy payments as non-executive medical and technical directors of OrganOx R Limited, and are shareholders. JF is the Chief Scientific Officer of the ex-vivo Research Center CIC, but receives no payments and has no shares and is an executive director of Perfusion Biotechnology Limited.

The remaining authors declare that the research was conducted in the absence of any commercial or financial relationships that could be construed as a potential conflict of interest.

Publisher's Note: All claims expressed in this article are solely those of the authors and do not necessarily represent those of their affiliated organizations, or those of the publisher, the editors and the reviewers. Any product that may be evaluated in this article, or claim that may be made by its manufacturer, is not guaranteed or endorsed by the publisher.

Copyright (c) 2022 Weissenbacher, Stone, Lo Faro, Hunter, Ploeg, Coussios, Fildes and Friend. This is an open-access article distributed under the terms of the Creative Commons Attribution License (CC BY). The use, distribution or reproduction in other forums is permitted, provided the original author(s) and the copyright owner(s) are credited and that the original publication in this journal is cited, in accordance with accepted academic practice. No use, distribution or reproduction is permitted which does not comply with these terms. 